\title{
Two novel STK11 missense mutations induce phosphorylation of S6K and promote cell proliferation in Peutz-Jeghers syndrome
}

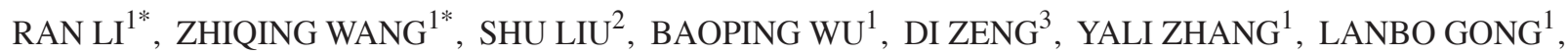 \\ FEIHONG DENG ${ }^{1}$, HAOXUAN ZHENG ${ }^{1}$, YADONG WANG ${ }^{1}$, CHUDI CHEN ${ }^{1}$, JUNSHENG CHEN ${ }^{1}$ and BO JIANG ${ }^{1,4}$ \\ ${ }^{1}$ Guangdong Provincial Key Laboratory of Gastroenterology, Department of \\ Gastroenterology, Nanfang Hospital, Southern Medical University, Guangzhou, Guangdong 510515; \\ ${ }^{2}$ Medical Genetics Center, Guangdong Women and Children's Hospital, Guangzhou, Guangdong 510010; \\ ${ }^{3}$ Department of Gastroenterology, Guangzhou Panyu Central Hospital, Guangzhou, Guangdong 511400; \\ ${ }^{4}$ Department of Gastroenterology, Beijing Tsinghua Changgung Hospital, Beijing 102218, P.R. China
}

Received February 19, 2016; Accepted January 19, 2017

DOI: $10.3892 / \mathrm{ol} .2017 .7436$

\begin{abstract}
Peutz-Jeghers syndrome (PJS) is a rare hereditary disease caused by mutations in serine threoninekinase 11 (STK11) and characterized by an increased risk of developing cancer. Inactivation of STK11 has been associated with the mammalian target of rapamycin (mTOR) pathway. Hyperactivation and phosphorylation of the key downstream target genes ribosomal protein S6 kinase 1 (S6K1) and S6 promote protein synthesis and cell proliferation. To better understand the effects of STK11 dysfunction in the pathogenesis of PJS, genomic DNA samples from 21 patients with PJS from 11 unrelated families were investigated for STK11 mutations in the present study. The results revealed 6 point mutations and 2 large deletions in $8(72.7 \%, 8 / 11)$ of the unrelated families. Notably, 3 novel mutations were identified, which included 2 missense mutations [c.88G >A (p.Asp30Asn) and c.869T>C (p.Leu290Pro)]. Subsequent immunohistochemical analysis revealed staining for phosphorylated-S6 protein in colonic hamartoma and breast benign tumor tissues from patients with PJS carrying
\end{abstract}

Correspondence to: Dr Zhiqing Wang, Guangdong Provincial Key Laboratory of Gastroenterology, Department of Gastroenterology, Nanfang Hospital, Southern Medical University, 1838 North Guangzhou Avenue, Guangzhou, Guangdong 510515, P.R. China E-mail:wzq2001ing@163.com

Professor Bo Jiang, Department of Gastroenterology, Beijing Tsinghua Changgung Hospital, 168 Li Tang Road, Changping, Beijing 102218, P.R. China

E-mail: drjiang@163.com

${ }^{*}$ Contributed equally

Abbreviations: PJS, Peutz-Jeghers syndrome; STK11, serine threonine kinase 11; mTOR, mammalian target of rapamycin; S6K1, ribosomal protein S6 kinase 1

Key words: Peutz-Jeghers syndrome, novel serine threonine kinase 11 mutants, cell proliferation, S6 kinase 1, S6 the two respective missense mutations. Additionally, the novel missense STK11 mutants induced phosphorylation of S6K1 and S6, determined using western blot analysis, and promoted the proliferation of HeLa and SW1116 cells, determined using Cell Counting Kit-8 and colony formation assays. Collectively, these findings extend the STK11 mutation spectrum and confirm the pathogenicity of two novel missense mutations. This study represents a valuable insight into the molecular mechanisms implicated in the pathogenesis of PJS.

\section{Introduction}

Peutz-Jeghers Syndrome [PJS; Mendelian Inheritance in Man (MIM), 175200, www.ncbi.nlm.gov/OMIM] is a rare autosomal dominant disorder, which is characterized by gastrointestinal hamartomatous polyps, mucocutaneous pigmentation and an elevated risk of various neoplasms $(1,2)$. Mutations in the serine threonine kinase 11/liver kinase B1 (STK11/LKB1; MIM, 602216) gene on chromosome 19p13.3 have been identified as the major cause of PJS (3-5).

Previously, direct sequencing of the STK11 gene in combination with a multiplex ligation-dependent probe amplification (MLPA) assay for deletion detection resulted in a mutation detection rate of $67.3 \%$ (35/52) in a group of PJS patients (6). This rate is consistent with the 50 to $90 \%$ frequency reported by various research groups $(3,7)$. The majority of mutations have been shown to be either frameshift or nonsense, resulting in an abnormally truncated protein and a subsequent loss of kinase activity (5). However, a number of STK11 missense mutations have been identified in PJS with unknown pathogenicity $(5,8)$.

STK11 has been implicated as an important regulator of cell proliferation and apoptosis $(8,9)$ via multiple signaling pathways (10), which may involve its tumor suppressor function and/or catalytic activity (11). The mammalian target of rapamycin (mTOR) pathway is one of the major downstream pathways that can be regulated by $S T K 11$ (12-14). The ribosomal protein S6 kinase 1 (S6K1) is the first mTOR substrate and has been an extensively studied effector of mTOR complex 1 (mTORC1) $(15,16)$. Furthermore, S6K1 activation requires mTORC1-mediated phosphorylation, which acts to improve 
S6K1 activity towards its S6 substrate $(16,17)$. Signals from S6K1 activation are involved in a variety of cellular functions, including the regulation of protein translation, cell growth, angiogenesis and metabolism $(18,19)$. Therefore, mutations in STK11 that inactivate its endogenous activity can negatively regulate mTORC1 signaling, resulting in phosphorylation and activation of its downstream targets. In turn, this is able to relieve inhibition on protein synthesis, and promote cell growth and tumorigenesis (20-22).

In the present study, STK11 genetic mutations were analyzed from PJS patients in 11 unrelated families, and $8(72.7 \%, 8 / 11)$ different mutations were found, including 6 point mutations and 2 large deletions. Notably, 3 of the mutations were novel. Of these 3 mutations identified, 2 were missense mutations [c.88G $>$ A (p.Asp30Asn) and c.869T $>C$ (p.Leu290Pro)]. Furthermore, the missense mutations were able to disrupt the function of the tumor suppressor STK11, which led to phosphorylation of S6K1 and S6 and promoted protein synthesis and cell proliferation.

These findings demonstrated the pathogenicity of the two novel missense mutations and extended the current understanding of STK11 mutations. Understanding how the function of STK11 is disrupted may lead to an improved understanding of the molecular mechanisms implicated in the pathogenesis of PJS and carcinogenesis. This may also provide potential therapeutic targets for future treatment.

\section{Materials and methods}

Patients. Peripheral blood samples of PJS patients $(\mathrm{n}=21)$ from 6 PJS families and 5 sporadic cases were collected between January 2012 and September 2015 at Nanfang Hospital (Guangzhou, China). The patients were diagnosed according to previously published criteria (6). Endoscopic or surgical polypectomy and histopathological examination (by $\geq 2$ pathologists) were performed in all patients. Endoscopic or surgical polypectomy were performed in all patients. The tissues were fixed in $10 \%$ formalin solution for $24 \mathrm{~h}$ at $4^{\circ} \mathrm{C}$ and embedded in paraffin for $0.5 \mathrm{~h}$ at $65^{\circ} \mathrm{C}$. Serial sections $(4 \mu \mathrm{m})$ were cut and prepared for hematoxylin-eosin (HE) staining and immunohistochemistry, as previously described (6). All HE-stained sections were reviewed by two experienced pathologists independently, who defined the hamartomatous polyps. Surgical resection was performed on other organs, including the breasts and cervix, in 3 patients due to the presence of benign or malignant tumors. The family cancer history was collected from the patients' medical records. All patients provided informed consent for this study, and the principles outlined in the Declaration of Helsinki were followed. Ethical approval was obtained from the Medical Ethics Committee at Nanfang Hospital.

Detection of STK11 germline mutations. Genomic DNA was extracted from peripheral blood using the commercially available QiAamp DNA Blood Midi kit (Qiagen $\mathrm{GmbH}$, Hilden, Germany). PCR primers were designed using the software program Primer (version 3.0; http://frodo.wi.mit. edu/primer3/) to amplify the STK11 exons (RefSeq accession number NM_000455.4) and intron-exon boundaries. Sanger sequencing was performed for each index case in the 6 familial and 5 sporadic cases, as previously described (6).
All STK11 exons (exon1-9) were amplified (for primers see Table I) using regular Taq polymerase (Takara Ex Taq; Takara Bio, Inc., Otsu, Japan). PCR amplification comprised an initial denaturation cycle at $95^{\circ} \mathrm{C}$ for $15 \mathrm{~min}$, followed by 38 amplification cycles consisting of $95^{\circ} \mathrm{C}$ for $30 \mathrm{sec}$, annealing at $60^{\circ} \mathrm{C}$ for $45 \mathrm{sec}$, and extension at $72^{\circ} \mathrm{C}$ for $7 \mathrm{~min}$. Sanger sequencing was performed for each index case in the 6 familial and 5 sporadic cases, as previously described (6). In familial cases, identified mutations were further tested in all available family members to confirm segregation of the mutation with the disease. All variants identified were screened against the single nucleotide polymorphism database (www.ncbi.nlm.nih. gov/SNP) to exclude the possibility of these variants being polymorphisms.

An MLPA assay was performed for large intragenic deletions using the MLPA test kit (SALSA P101-B1 STK11; MRC-Holland, Amsterdam, Netherlands) as previously described (6). Deletion screening was performed according to the manufacturer's instructions, and the results were analyzed using the GeneMarker ${ }^{\circledR}$ HID STR Human Identity software (version 3.0; SoftGenetics, LLC., State College, PA, USA). Values of 0.85 to 1.15 indicate normal results (presence of two copies), and values of 0.35 to 0.65 or 1.35 to 1.65 indicate a deletion or duplication, respectively. All identified deletions were confirmed in a second independent reaction and confirmed to segregate within the relevant family members.

Predicting the effect of the identified mutations. The novel missense mutations were all predicted using the PolyPhen-2 web tool (PolyPhen2: Http://genetics.bwh.harvard.edu/pph2/). The PolyPhen-2 score represents the probability that a substitution is damaging. The novel missense mutation (p.Leu290Pro) is predicted to be highly likely to be damaging with a score of 1.0, and the other (p.Asp30Asn) is predicted to be possibly damaging with a score of 0.775 . Variants with scores of 0.0 are predicted to be benign. Values closer to 1.0 are more confidently predicted to be deleterious.

Tissue staining. Immunohistochemistry (IHC) was performed as previously described (6). Tissue sections were deparaffinized and incubated with a phosphorylated (p)-S6 (Ser240/244) primary antibody (1:400; catalog no. 2903; Cell Signaling Technology, Inc., Danvers, MA, USA) overnight at $4^{\circ} \mathrm{C}$. After three washes with PBST $(2,000 \mathrm{ml} \mathrm{PBS}+4 \mathrm{ml}$ Tween 20), the sections were incubated with biotin-conjugated goat anti-rabbit immunoglobulin G (1:300; catalog no. SPN-9001; Invitrogen; Thermo Fisher Scientific, Inc.) for $10 \mathrm{~min}$ at room temperature, and then incubated with streptavidin-peroxidase for $10 \mathrm{~min}$ at room temperature. A known p-S6 positive colorectal cancer tissue, from one patient who had undergone endoscopic at Nanfang Hospital. HE stained specimens were reviewed by a senior pathologist, who determined the polyp histology and tumor type, was used as a positive control for p-S6 staining. p-S6 staining was scored as positive when $>10 \%$ of the cells exhibited nuclear expression.

Reverse transcription-quantitative polymerase chain reaction $(R T-q P C R)$. Total RNA was extracted using Trizol (Invitrogen; Thermo Fisher Scientific, Inc., Waltham, MA, USA), and cDNA was synthesized by reverse transcription from $1 \mu \mathrm{g}$ 
total RNA using oligo(dT) primers (Promega Corporation, Madison, WI, USA). RT-qPCR was performed using the All-in-One ${ }^{\mathrm{TM}}$ qPCR mix (GeneCopoeia Inc., Rockville, MD, USA) on a LightCycler 480 system (Roche Diagnostics, Basel, Switzerland). Primer sequences for STK11 were as follows: Forward, 5'-AGGGCCGTCAAGATCCTCAA-3' and reverse, 5'-GCATGCCACACACGCAGTA-3'. Primer sequences for glyceraldehyde-3-phosphate dehydrogenase (GAPDH) were as follows: Forward, 5'-GAAGGTGAAGGTCGGAGTC-3' and reverse, 5'-GAAGATGGTGATGGGATTTC-3'. The housekeeping gene GAPDH was used as an internal control for normalization. The relative expression of STK11 was calculated using the $2^{-\Delta \Delta \mathrm{Cq}}$ method (23). Experiments were repeated three times and the mean of the data was determined.

Western blotting. Total protein was extracted using radioimmunoprecipitation assay lysis buffer with protease and phosphatase inhibitors (both, 1:100; Roche, Nutley, NJ, USA). Total protein was quantified using bicinchoninic acid assay (Thermo Fisher Scientific, Inc.). Total protein $(25 \mu \mathrm{g})$ was resolved by $10 \%$ SDS-PAGE (Bio-Rad Laboratories, Inc., Hercules, CA, USA) and transferred to polyvinylidene difluoride membranes (EMD Millipore, Billerica, MA, USA). Membranes were blocked with 5\% BSA (Bio-Rad Laboratories, Inc.) in PBS with $0.1 \%$ Tween-20 for $2 \mathrm{~h}$ at room temperature. Membranes were incubated with primary antibodies against STK11 (1:1,000; catalog no. 3047) S6K1 (1:1,000; catalog no. 2903) S6 (1:1,000; catalog no. 1155), p-S6K1 (Thr389; 1:1,000; catalog no. 9234) and p-S6 (Ser240/244; 1:1,000; catalog no. 2903) (all Cell Signaling Technology, Inc.) overnight at $4^{\circ} \mathrm{C}$. Mouse polyclonal anti-GAPDH antibody (1:3,000; Santa Cruz Biotechnology, Inc., Dallas, TX, USA) was used as the loading control. The membranes were subsequently incubated for $2 \mathrm{~h}$ at room temperature with either horseradish peroxidase (HRP)-conjugated goat anti-rabbit $\operatorname{IgG}(1: 2,000$; catalog no. 2903; Cell Signaling Technology, Inc.) or HRP-conjugated goat anti-mouse IgG (1:5,000; catalog no. sc-2005; Santa Cruz Biotechnology, Inc.). Protein expression was detected using an enhanced chemiluminescence kit (Thermo Fisher Scientific, Inc.).

Cell lines and culture. Human colon cancer (SW1116, HT29, SW620, SW480, LoVo, Caco2, and HCT116), human cervical carcinoma (HeLa), normal human gastric epithelial (GES-1), and human kidney (293T) cell lines were cultured in Dulbecco's modified Eagle's medium (Gibco; Thermo Fisher Scientific, Inc.) supplemented with $10 \%$ fetal bovine serum (Gibco; Thermo Fisher Scientific, Inc.) and maintained in a humidified atmosphere of $5 \% \mathrm{CO}_{2}$ at $37^{\circ} \mathrm{C}$. All cell lines used in the present study were obtained from the American Type Culture Collection, Rockville, MD, USA.

Cell lines transfection. SW117 and HeLa cells were used for transfection and subsequent experiments. The sequences of all STK11 gene mutant plasmids were confirmed by genetic sequencing. The constructs pGCMV-green fluorescent protein (GFP) (empty vector), pGCMV-GFP-STK11 WT (wild-type), pGCMV-GFP-STK11 p.Leu290Pro and pGCMV-GFP-STK11 p.Asp30Asn were synthesized by Shanghai GenePharma Co., Ltd., (Shanghai, China). Cells were seeded $\left(4 \times 10^{5}\right)$ in tissue culture plates and grown to 70 to $80 \%$ confluence. Subsequently, the cells were transfected with the vectors using Lipofectamine 3000 (Invitrogen; Thermo Fisher Scientific, Inc.) according to the manufacturer's instructions. Gene overexpression was examined by RT-qPCR, and protein expression was analyzed by western blotting at 24 to $48 \mathrm{~h}$ post-transfection. Stable transfections were performed at $48 \mathrm{~h}$ post-transfection, and the transfected cells were grown with G418 (400 mg/ml; Sigma-Aldrich; Merck Millipore, Darmstadt, Germany) for 4 to 8 weeks to allow for positive selection.

Cell Counting Kit-8 (CCK-8) and colony formation assays. To determine the effect of novel STK11 mutants on cell proliferation, transfected cells were measured using CCK-8 (Dojindo Molecular Technologies, Inc., Kumamoto, Japan) according to the manufacturer's protocols. Proliferation was assessed by colony formation assay. Transfected cells were counted and seeded into 6-well plates (1,000 cells/well). The number of colonies containing $>50$ cells were stained with $0.5 \%$ crystal violet in isopropanol and counted 2 weeks after seeding. The relative cell colony numbers were evaluated as the ratio of the last visible colonies to those at initialization.

Flow cytometric analysis. Cell apoptosis was quantitated using the Annexin V-APC and 7-aminoactinomycin D (7-AAD) double dye cell apoptosis kit (BD Biosciences, Franklin Lakes, NJ, USA) according to the manufacturer's protocol. Cells $\left(1 \times 10^{6}\right.$ cells/well) were collected at $48 \mathrm{~h}$ post-transfection with the aforementioned STK11 plasmids and washed twice with ice-cold PBS. The cells were re-suspended in $500 \mu 11 \mathrm{X}$ binding buffer, stained with $5 \mu \mathrm{l}$ Annexin V-APC and $5 \mu \mathrm{l}$ $7-\mathrm{AAD}$ and incubated on ice for $15 \mathrm{~min}$ in the dark. Apoptotic cells $(10,000$ cells/test) were quantified by flow cytometer (BD Biosciences).

Statistical analysis. All statistical analyses were performed using SPSS (version 17.0; SPSS Inc., Chicago, IL, USA). The data are expressed as the mean \pm standard deviation unless otherwise noted. Comparisons among multiple groups were determined using one-way analysis of variance, followed by least significance difference or Dunnett's T3 test. $\mathrm{P}<0.05$ was considered to indicate a statistically significant difference.

\section{Results}

Identification of STK11 mutations. The present study identified 6 different germline point mutations in 6 out of 11 index cases (52.4\%; Table II). A number of mutations $(3 / 6,50 \%)$ were associated with colon or esophagus cancer in either index patients or in relatives with PJS. A total of 4 mutations were identified in familial cases $(4 / 6,66.7 \%)$ and 2 in sporadic cases $(2 / 5,40 \%)$ of PJS. Of the 6 mutations identified, 4 were truncation mutations and 2 were missense mutations. To the best of our knowledge, 3 of the mutations [c.143_144insA (p.Try49Valfs*114), c.869T>C (p.Leu290Pro) and c.88G >A (p.Asp30Asn)] have previously been unreported and are therefore novel (Fig. 1; Table II).

MLPA was used to determine the presence or absence of exonic rearrangements in the 5 PJS probands, where no 
Table I. The polymerase chain reaction primers of 9 exons of STK11 gene.

\begin{tabular}{|c|c|}
\hline Primer & Primer sequence $\left(5^{\prime}-3^{\prime}\right)$ \\
\hline \multirow[t]{2}{*}{ Exon 1A } & F: CTCAGGGCTGGCGGCGGGACT \\
\hline & R: CTTGCGGCGCGGCTGGTAGATGA \\
\hline \multirow[t]{2}{*}{ Exon 1B } & F: ACGTTCATCCACCGCATCGAC \\
\hline & R: GCACAGCGTCTCCGAGTCCAG \\
\hline \multirow[t]{2}{*}{ Exon1C } & F:TCTTACGGCAAGGTGAAGGAGGTG \\
\hline & R: CCGACCCCAGCAAGCCATACTTA \\
\hline \multirow[t]{2}{*}{ Exon 2} & F: ATACACCCCTGTCCTCTCTG \\
\hline & R: AGGCCCCGCGGTCCCAAC \\
\hline \multirow[t]{2}{*}{ Exon 3} & F: CTGAGCTGTGTGTCCTTAGCG \\
\hline & R: GTGTGGCCTCACGGAAAGGAG \\
\hline \multirow[t]{2}{*}{ Exon 4} & F: CGGCCCCAGGACGGGTG \\
\hline & R: GTGCAGCCCTCAGGGAG \\
\hline \multirow[t]{2}{*}{ Exon 5} & F: ACTCCCTGAGGGCTGCAC \\
\hline & R: CCCCTCGGAGTGTGCGTGTGG \\
\hline \multirow[t]{2}{*}{ Exon 6} & F: TCAACCACCTTGACTGACCA \\
\hline & R: ACACCCCCAACCCTACATTT \\
\hline \multirow[t]{2}{*}{ Exon 7} & F: TCACCCAGGGCCTGACAACAGAG \\
\hline & R: GCAGCCTCGGCCCCACTG \\
\hline \multirow[t]{2}{*}{ Exon 8} & F: GGCGCCACTGCTTCTGGGC \\
\hline & R: CGAGTCAGCAGAGCCGGGC \\
\hline \multirow{2}{*}{ Exon 9A } & F: TGTAAGTGCGTCCCCGTGGTG \\
\hline & R: CGCCCTGGATTTGGTGCTA \\
\hline \multirow[t]{2}{*}{ Exon 9B } & F: GTGTATGAACGGCACAGAGGC \\
\hline & R: CAGGCGTTGTCCCCACAT \\
\hline
\end{tabular}

mutations were identified by Sanger sequencing. This resulted in the identification of two large deletions, with one being associated with the presence of breast cancer in the relative of the proband (PJS7; Table II). The two deletions were predicted to affect the kinase domain of the protein. The total mutation detection rate in the index cases was $72.7 \%$ (8/11), which indicated that genetic alterations in STK11 in individuals with PJS may increase the risk of developing PJS-associated cancer.

In total, 3 of the truncation mutations identified in the present study have previously been described $(7,24,25)$, and 1 is novel [c.143_144insA (p.Try49Valfs*114)] (PJS2; Table II). The novel mutation involves an insertion of nucleotide $\mathrm{A}$ between nucleotides 143 and 144 in exon 1. This results in a 433-amino acid sequence with a frameshift after nucleotide 49 and premature termination at nucleotide 114. Therefore, the alteration leads to a complete loss of the kinase domain of STK11 (26).

The novel missense mutation [c.869T $>\mathrm{C}$ (p.Leu290Pro)] leads to an amino acid change at codon 290 in exon 7, which is located in the catalytic kinase region of STK11 (PJS4 II-3; Fig. 1C; Table II). The other novel missense mutation [c.88G $>$ A (p.Asp30Asn)] leads to an alteration at nucleotide $88(\mathrm{G}<\mathrm{A})$ and an amino acid change at codon 30 in exon 1, which is located at the N-terminal of STK11 (PJS5 III-2; Fig. 1D; Table II). This results in the substitution of aspartate by asparagine. The two mutations were predicted to be pathogenic by PolyPhen2 (http://genetics.bwh.harvard.edu/pph2/).

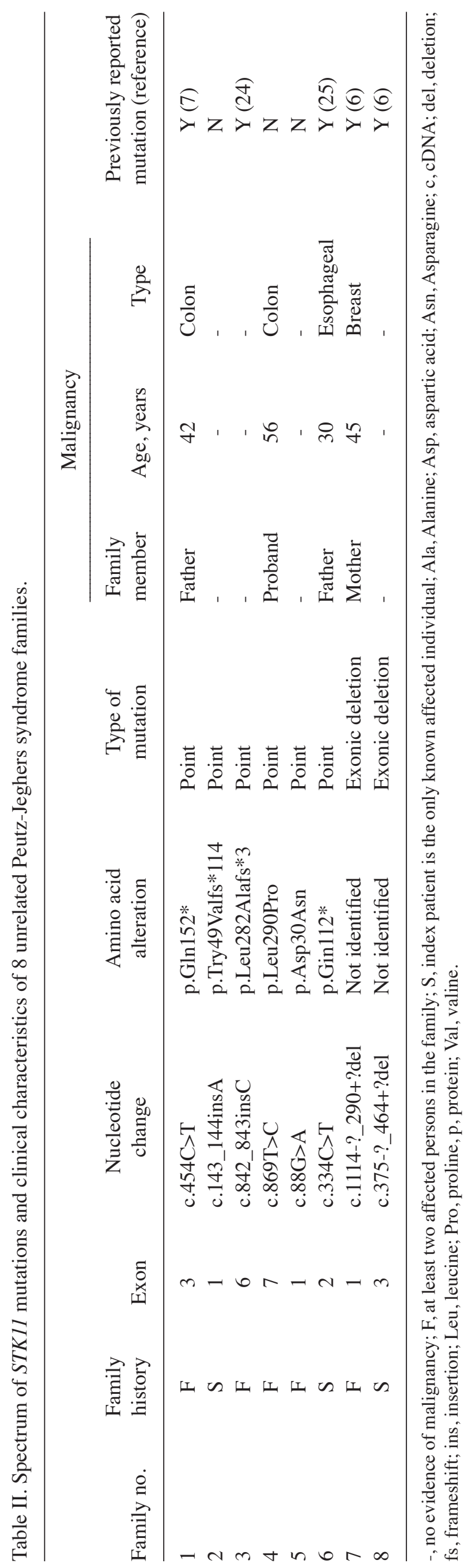


A

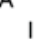

II

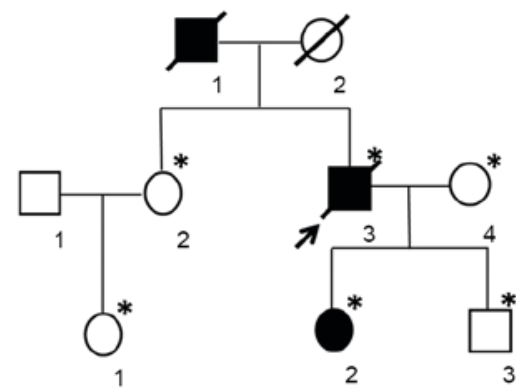

C

WT

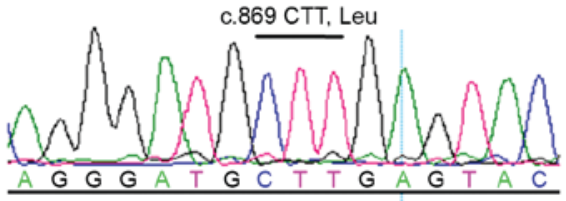

MUT

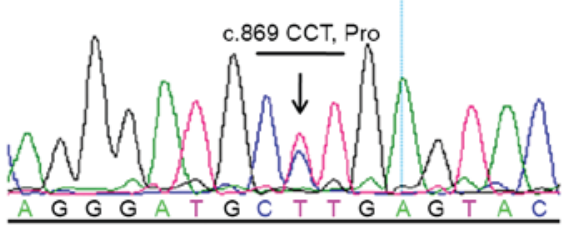

B

।

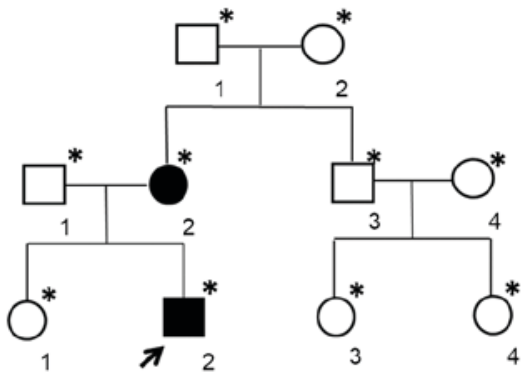

WT

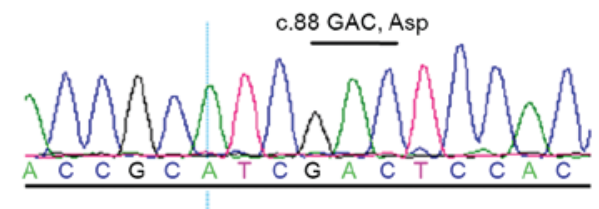

MUT

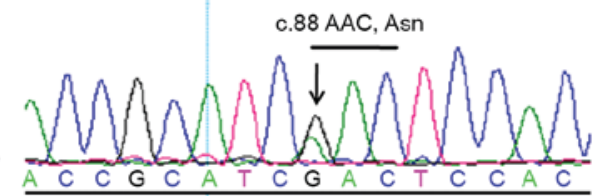

Figure 1. Segregation of 2 novel STK11 missense mutations in 2 PJS families. Pedigree charts in 2 unrelated families with PJS (PJS4 and PJS5). (A) PJS4 II-2, PJS4 II-4, PJS4 III-1 and PJS4 III-3 carried the wild-type c.869T (p.Leu290); while PJS4 III-2 carried the mutation c.869T>C (p.Leu290Pro). Proband (PJS4 II-3) carried the mutation c.869T>C (p.Leu290Pro) and succumbed to colon adenocarcinoma at 56 years of age. (B) PJS5 I-1, PJS5 I-2, PJS5 II-1, PJS5 II-3, PJS5 II-4, and PJS5 III-1, PJS5 III-3, PJS5 III-4 carried the wild-type c.88G (p.Asp30); while PJS5 II-2 and PJS5 III-2 carried the mutation c.88G >A (p.Asp30Asn). Arrow, proband; square, males; circle, females; open symbols, unaffected individuals; solid symbols, affected individuals; diagonal line, deceased; "genotypes of individuals examined by DNA analysis. Sequencing chromatograms of genomic DNA from the proband (C) PJS4 II-3 and (D) PJS5 III-2. Arrows indicate the position of the mutation and the codon containing the mutation is underlined. Asp, aspartic acid; Asn, Asparagine; Leu, leucine; MUT, mutated; PJS, Peutz-Jeghers syndrome; Pro, proline; STK11, serine threonine kinase 11; WT, wild-type.

Novel STK11 missense mutations induce p-S6 expression in tissues from PJS patients. To investigate whether the novel missense mutations affect the phosphorylation of mTOR pathway key downstream target gene $S 6$, IHC was performed to detect p-S6 (Ser240/244) expression in colon hamartomatous polyp and colorectal mucosal epithelium tissues obtained from individuals with or without STK11 mutations. p-S6 staining was observed in the nuclei of epithelial cells in colonic hamartoma obtained from patient PJS4 II-3 with the mutation c.869T $>$ C (p.Leu290Pro) (Fig. 2B) and in the breast intraductal papillary tumor from the patient PJS5 III-2 with the mutation c.88G $>$ A (p.Asp30Asn) (Fig. 2E). By contrast, p-S6 staining was not observed in colorectal mucosa derived from PJS4 III-3, who is a relative of PJS4 II-3 (Fig. 2C) and in breast hyperplasia glandular epithelial obtained from PJS5 II-2, who is a relative of PJS5 III-2 (Fig. 2F). PJS4 III-3 and PJS5 II-2 did not carry STK11 mutations. These findings suggest that the two novel missense mutations may disrupt the protein function of STK11 serine/threonine kinase and induce S6 phosphorylation.

Expression of novel STK11 mutations in HeLa and SW1116 cells. RT-qPCR and western blotting were used to detect endogenous expression levels of STK11 in human cell lines. As shown in Fig. 3A, STK11 expression levels were nearly undetectable in HeLa and SW1116 cells when compared with all other tested cell lines. Subsequently, HeLa and SW1116 cells were used for further studies, and the effects of the novel STK11 mutations were investigated.
STK11 plasmids (empty vector, wild-type vector, STK11 mutant p.Leu290Pro vector and STK11 mutant p.Asp30Asn vector) were transfected into HeLa and SW1116 cells, and STK11 expression was examined by RT-qPCR and western blot analysis. As shown in Fig. 3B and C, the expression of STK11 in HeLa and SW1116 cells transfected with the STK11 mutants and the wild-type vector was significantly increased when compared with cells transfected with the empty vector. However, there were no significant differences in STK11 expression in cells transfected with the wild-type vector and the STK11 mutants. Taken together, these results suggest that the novel STK11 mutants have negligible effects on STK11 transcription and translation.

Novel STK11 missense mutations induce phosphorylation of S6K1 and S6 in cell lines. The effects of the two novel STK11 missense mutations on gene function and whether the mutations result in dysregulation of the downstream mTOR-mediated pathway was investigated. S6K1 and S6 are substrates of mTORC1. Phosphorylation of S6K1 at Thr389 and S6 at Ser240/244 was analyzed using western blotting. As shown in Fig. 4, there was increased expression of p-S6K1 (Thr389) and p-S6 (Ser240/244) in HeLa and SW1116 cells transfected with the two novel missense STK11 mutants compared with cells transfected with the empty vector. By contrast, cells transfected with the wild-type vector exhibited a marked decrease in the levels of p-S6K1 and p-S6. These results demonstrated that the novel STK11 missense mutations disrupted the endogenous protein kinase activity of STK11, which led to the activation of the mTORC1 signaling pathway. Consequently, this results in 

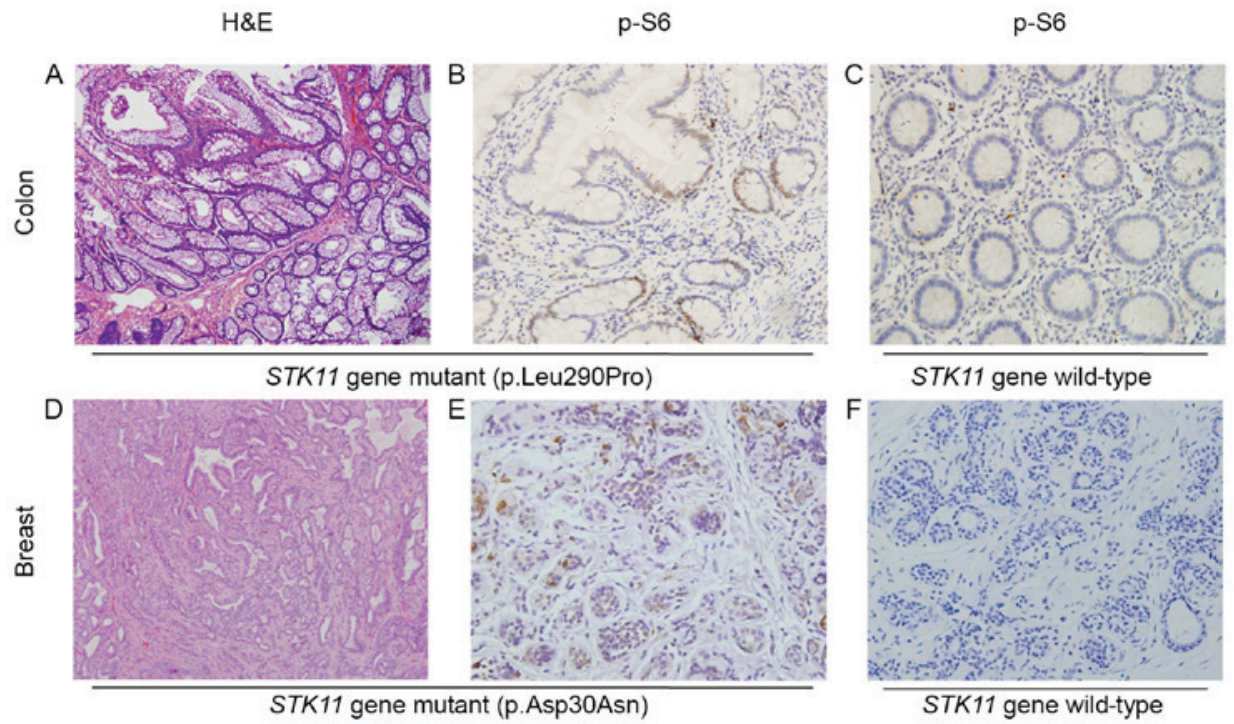

Figure 2. Staining of tissue sections from patients with PJS. (A) Hematoxylin and eosin staining of a colon hamartomatous polyp obtained from the patient PJS4 II-3 carrying the STK11 missense mutation c.869T>C (p.Leu290Pro). Micrograph shows branched bundles of smooth muscle fibers. Magnification, x100. (B) Immunohistochemical staining of a colon hamartomatous polyp obtained from patient PJS5 III-2 with the STK11 missense mutation c.88G $>$ A (p.Asp30Asn). Image shows the localization of p-S6 (Ser240/244) to the epithelial cell nuclei. Magnification, x400. (C) p-S6 staining is absent in the colorectal mucosa epithelium samples without STK11 mutations. Magnification, x400. (D) Hematoxylin and eosin staining of breast intraductal papillary tumor tissues obtained from patient PJS5 III-2. Image shows marked cellular heterogeneity in pathology. Magnification, x100. (E) Immunohistochemical staining of breast intraductal papillary tumor tissues obtained from patient PJS5 III-2. Image shows localization of p-S6 to the nuclei of glandular epithelial cells. Magnification, x400. (F) p-S6 staining is absent in the ductal epithelium of breast hyperplasia samples without STK11 mutations. Magnification, x400. Asp, aspartic acid; Asn, Asparagine; Leu, leucine; PJS, Peutz-Jeghers syndrome; p, phosphorylated; Pro, proline; STK11, serine threonine kinase 11.

A
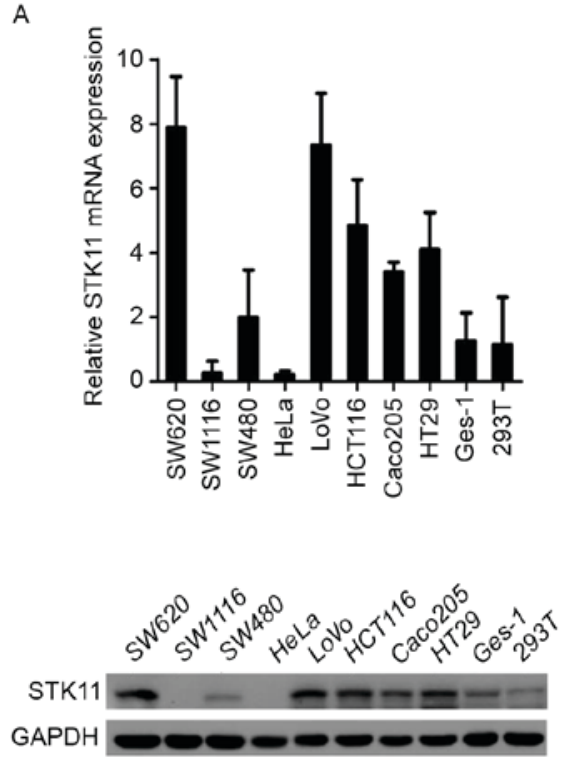

B

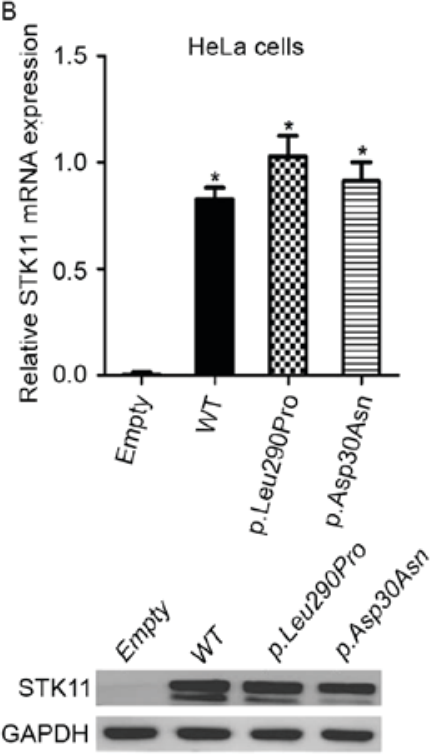

C.
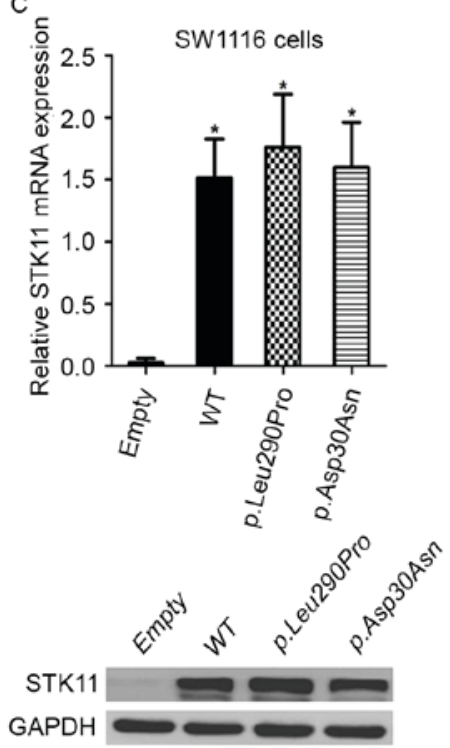

Figure 3. Expression of STK11 in human cell lines and STK11 mutants in HeLa and SW1116 cells. (A) Relative STK11 mRNA expression levels were markedly reduced in SW1116 and HeLa cells. (B) STK11 protein expression in HeLa and SW1116 cells was not detected. mRNA and protein expression levels were significantly increased in (C) HeLa and (D) SW1116 cell lines transfected with WT, STK11 mutant p.Leu290Pro and STK11 mutant p.Asp30Asn vectors compared with cells transfected with the empty vector. No significant differences between the WT vector and the STK11 mutant vectors were observed. "P<0.05. Asp, aspartic acid; Asn, Asparagine; GAPDH, glyceraldehyde 3-phosphate dehydrogenase; Leu, leucine; PJS, Peutz-Jeghers syndrome; p, phosphorylated; Pro, proline; STK11, serine threonine kinase 11; WT, wild-type.

the dysregulation of the STK11-S6K1 axis. We hypothesized that this disruption leads to abnormal de-repression of protein synthesis and promotion of cell growth.

Cell proliferation is promoted by two novel STK11 missense mutations. To further determine whether the novel missense
STK11 mutations are able to effectively suppress cell growth and proliferation, CCK-8 and colony formation assays were performed. As shown in Fig. 5, HeLa and SW1116 cells transfected with wild-type vectors exhibited a marked reduction in cell growth compared with cells transfected with the STK11 mutant vectors. The growth of HeLa and SW1116 


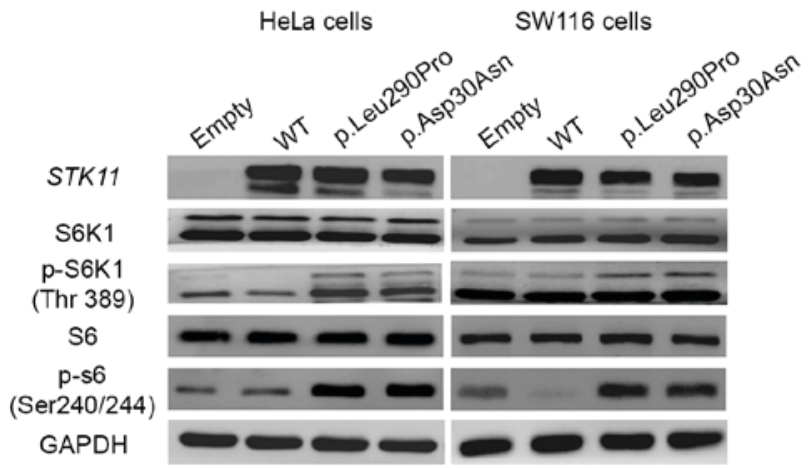

Figure 4. Missense STK11 mutations [c.869T>C (p.Leu290Pro) and c.88G >A (p.Asp30Asn)] induce phosphorylation of S6K1 and S6. Western blotting of key mTOR-mediated target genes in transfected cells. Expression of p-S6K1 and p-S6 in cells transfected with STK11 mutant p.Leu290Pro and STK11 mutant p.Asp30Asn vectors increased compared with cells transfected with empty vectors and WT vectors. Total levels of S6K1 and S6 remained unchanged. Asp, aspartic acid; Asn, Asparagine; GAPDH, glyceraldehyde 3-phosphate dehydrogenase; GFP, green fluorescent protein; Leu, leucine; mTOR, mammalian target of rapamycin; p, phosphorylated; Pro, proline; Ser, serine; STK11, serine threonine kinase 11; S6K1, ribosomal protein S6 kinase 1; Thr, threonine; WT, wild-type.

cells transfected with the STK11 mutants (p.Leu290Pro and p.Asp30Asn) was significantly increased $(\mathrm{P}<0.01)$ when compared with the empty vector control (Fig. 5A and C). These results suggest that STK11 gene is a tumor suppressor gene in the two cell lines. The two novel STK11 mutations abrogated cellular growth suppression function of the STK11 gene, which contributes to tumorigenesis. Furthermore, the effect of the novel STK11 missense mutations on cell apoptosis was investigated. Compared with the empty vector control, the wild-type vector promoted apoptosis. However, there was no statistically significant difference between the two novel missense STK11 mutants and the control (data not shown). These findings indicate that although the STK11 gene exerts a role in promoting apoptosis in HeLa and SW1116 cells, the two novel STK11 missense mutations have no significant effect.

\section{Discussion}

In the present study, a cohort of 21 affected PJS patients were analyzed, which included index cases and family members. Direct sequencing of the STK11 gene combined with the MLPA assay for the detection of deletions resulted in a mutation detection rate of $72.7 \%$ (8/11). This rate is consistent with the $67.3 \%$ frequency reported previously (6). Notably, to the best of our knowledge, 3 of the mutations identified were novel, and therefore the present study has extended the spectrum of known disease-causing STK11 mutations.

The STK11 gene contains nine exons (27) encoding a 433-amino acid protein that has a single catalytic kinase domain for adenosine 5'-triphosphate binding, two N-terminal nuclear leading sequences that contain nuclear localization signals and a $\mathrm{C}$-terminal regulatory domain that contains a prenylation motif (CAAX-box) $(26,28)$. The catalytic kinase domain is located at nucleotides 44 to $309(26,28)$.

The novel PJS missense mutation, c.869T>C (p.Leu290Pro), identified in the proband PJS4 II-3 in the present study, leads to an amino acid alteration at codon 290 in exon 7, which is located in the catalytic kinase region of the STK11 protein. The proband was diagnosed with colon adenocarcinoma at 56 years of age. It has been reported that the majority of reported PJS-associated missense mutations are located in the STK 11 kinase domain and are crucial to the function of the STK11 protein $(3,7,29)$. These mutations may lead to the loss of inhibition on cell growth $(30,31)$, which suggests that c.869T $>\mathrm{C}$ may be pathogenic.

The other novel PJS missense mutation, c.88G $>$ A, identified in the proband PJS5 III-2, leads to an amino acid alteration at codon 30 in exon 1 located at the N-terminal of the STK11 protein. The proband PJS5 III-2 was diagnosed with a breast intraductal papillary tumor and has a family with a history of PJS. This change results in the substitution of aspartate by asparagine. The N-terminal of the STK11 protein contains a nuclear localization signal domain, which is required for the interaction with other proteins in order to be actively exported out of the nucleus $(30,31)$. The translocation of STK11 from the nucleus to the cytoplasm is associated with the activity of STK11 kinase, which is closely dependent on the formation of the STK11/Ste20-related adaptor/calcium-binding protein 39 complex $(29,31)$. Notably, a number of previously reported PJS mutants are retained in the nucleus $(3,32)$. This suggests that that the mutation, $\mathrm{c} .88 \mathrm{G}>\mathrm{A}$, may affect the translocation of STK11 from the nucleus to the cytoplasm, and therefore, it is predicted to be pathogenic. Consistent with the existing studies, a number of PJS patients carry germline mutations that inactivate the kinase activity of STK11 (33).

The inactivation of STK11 kinase activity has also been associated with an increased risk for the development of cancer (34). This relative cancer risk was estimated to be 9 to 18 times higher in PJS patients compared with the general population $(35,36)$. By contrast, Hearle et al (1) indicated that there is no significant correlation between the incidence of a tumor and its type from an analysis of clinical data of 419 patients with PJS.

The development of the PJS phenotype is speculated to be due to the loss of the kinase activity of STK11, which is associated with the loss of its growth suppression function (32). Therefore, mutations that affect the kinase activity of the STK11 protein are most likely to contribute to the development of the PJS phenotype and potentially to malignancy.

The tumor suppressor gene STK11 has an important role in cell proliferation through a multitude of targets, all of which may require the tumor suppressor function of this kinase and/or its catalytic activity $(8,11)$. Loss of $S T K 11$ is able to activate certain downstream signaling pathways to promote the growth and metastasis of tumors (14). Furthermore, it has been reported that mTOR is a central node that acts to integrate different cell signals to regulate cell growth (18). The kinase activity of STK11 protein primarily downregulates the mTORC1 signaling pathway and prevents the phosphorylation of the major downstream targets S6K1 and S6. S6K1 and S6 have been identified as important cell proliferation effectors, and phosphorylation of these targets leads to inhibition of cell proliferation and tumorigenesis $(10,12,13)$. Furthermore, it has been reported that phosphorylation of S6K1 leads to an increased number of hamartomatous gastrointestinal polyps in STK11 mutant mice $(21,37)$, suggesting that mTOR 

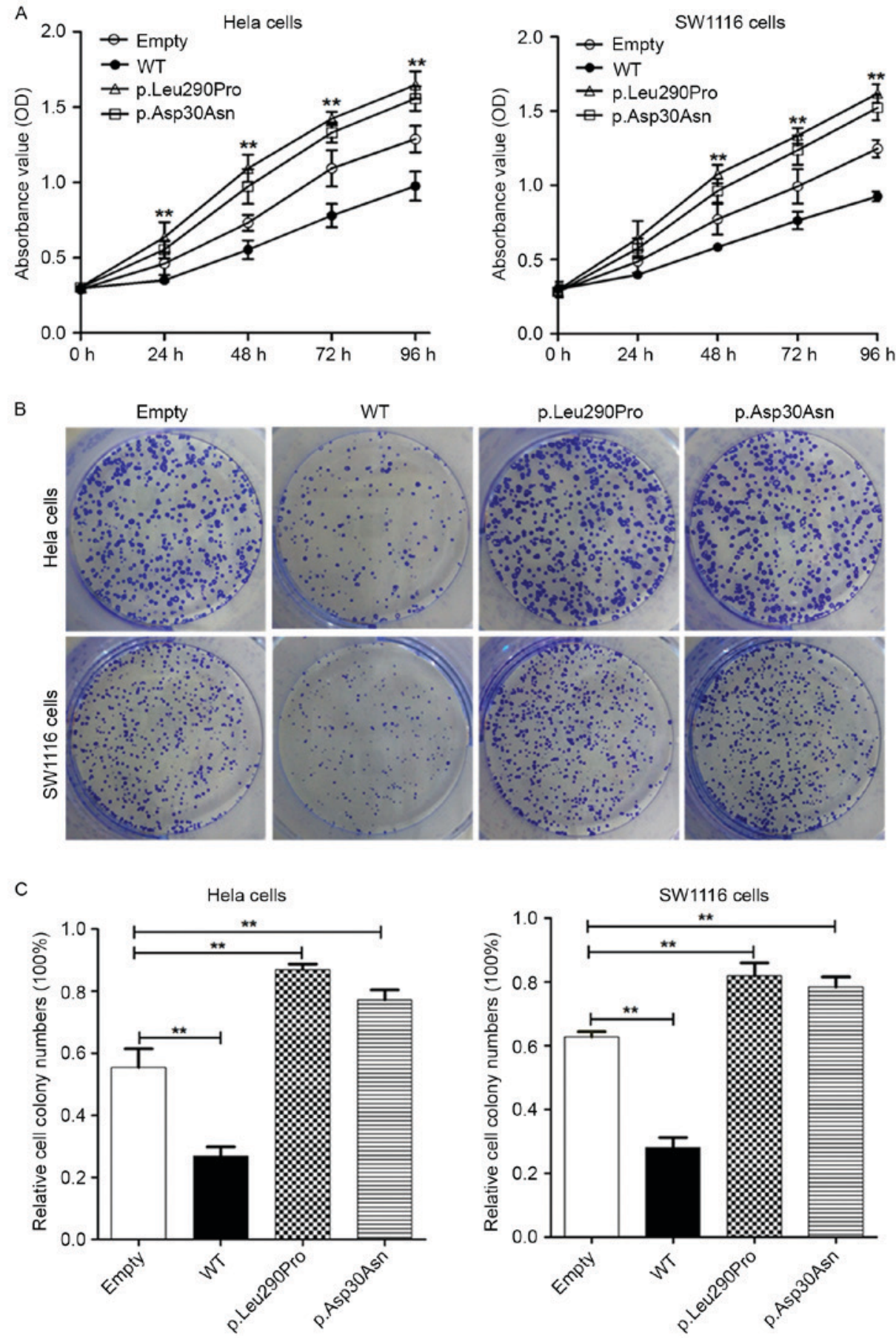

Figure 5. Missense STK11 mutations [c.869T>C (p.Leu290Pro) and c.88G>A (p.Asp30Asn)] promote proliferation of HeLa and SW1116 cells. (A) Cell counting kit-8 assay revealed that the proliferative capacity of cells transfected with STK11 mutant p. Leu290Pro vector was significantly increased compared with cells transfected with WT vectors. (B) Images of colony formation assays in HeLa and SW1116 cells transfected with empty vector, WT, STK11 mutant p.Leu290Pro and STK11 mutant p.Asp30Asn vectors. (C) Colony counts were evaluated by determining the ratio between HeLa and SW1116 cells transfected with empty, WT, STK11 mutant p.Leu290Pro or STK11 mutant p.Asp30Asn vectors. Statistical analysis was performed using a one-way analysis of variance between all groups. The data are represented as the mean values of three independent experiments. ${ }^{* *} \mathrm{P}<0.01$. Asp, aspartic acid; Asn, Asparagine; Leu, leucine; Pro, proline; STK11, serine threonine kinase 11; WT, wild-type.

overactivation contributes to hamartomatous tumor growth when STK11 is inactivated.

In the present study, immunohistochemical analysis of two novel missense STK11 mutant tissue samples exhibited an increased immunostaining signal for $\mathrm{p}-\mathrm{S} 6$. This finding suggests that inactivation of STK11 protein may result from novel missense mutations, leading to dysregulation of the mTOR signaling pathway and induction of S6 phosphorylation to promote protein synthesis and cell proliferation. In order to further investigate the effects of two novel missense mutations, cell viability was determined in vitro. The results indicated that the two novel STK11 missense mutants abrogated the tumor suppression function of the STK11 gene, and promoted cell growth and proliferation. In addition, western blotting demonstrated increased phosphorylation of S6K1 and S6 proteins. These results indicated that loss-of-function 
mutations in STK11 serine/threonine kinase are able to hyperactivate the STK11-mTORC1 axis, which leads to aberrant mTORC1-mediated phosphorylation of S6K1 and S6. This, in turn, promotes cell proliferation and malignant transformation $(21,38)$.

Furthermore, STK11 has also been found to suppress anti-apoptotic factors, including signal transducer and activator of transcription 3, c-Jun N-terminal kinase 1, c-Myc, k-Ras, mitogen-activated protein kinase and cyclooxygenase-2, to inhibit cell survival $(39,40)$. STK11 is able to induce cell apoptosis under metabolic stresses, including hypoxia and energy deprivation $(8,12)$. Previous studies reported the absence of STK11 staining and reduced numbers of apoptotic cells in polyps derived from PJS patients $(41,42)$. In the present study, it was demonstrated that transfection of the wild-type STK11 vector was able to promote cell apoptosis. The two novel STK11 missense mutations, located at the catalytic kinase region and the N-terminal of the $S T K 11$ protein, are associated with the impaired function of $S T K 11$ protein and are therefore pathogenic.

Although the detailed mechanisms of STK11 gene function as a tumor suppressor remains to be fully understood, STK11 has been shown to control cell growth via regulating mTOR-associated signaling pathways and cellular responses (18). For example, activation of mTORC1 stimulates angiogenesis by stabilizing hypoxia-inducible factor $1 \alpha$ under hypoxic conditions (43). Furthermore, activation of mTORC1 inhibits autophagy via phosphorylation of autophagy-related protein 13 and ULK1/2 (44). Rapamycin, a specific mTOR inhibitor, has been developed as an effective drug for the treatment of PJS and its associated tumors $(22,45)$.

Taken together, the two novel missense mutations of STK11 identified in the present study are able to disrupt the tumor suppression function of STK11 and impair STK11 serine/threonine protein kinase activity. Therefore, the major downstream molecules of the mTOR signaling pathway, S6K1 and S6, may be potential therapeutic targets for STK11-associated cancer.

\section{Acknowledgements}

The present study was supported by the Guangzhou Pilot Project of Clinical and the Translational Research Center (Early Gastrointestinal Cancers; grant no. 7415696196402), the Guangdong Provincial Bioengineering Research Center for Gastroenterology Diseases, the National Natural Science Foundation of China (grant no. 81401925), the Natural Science Foundation of Guangdong Province (grant no. 2015A030310102) and the President Foundation of Nanfang Hospital, Southern Medical University (grant no. 2013B005).

\section{References}

1. Hearle N, Schumacher V, Menko FH, Olschwang S, Boardman LA, Gille JJ, Keller JJ, Westerman AM, Scott RJ, Lim W, et al: Frequency and spectrum of cancers in the Peutz-Jeghers syndrome. Clin Cancer Res 12: 3209-3215, 2006.

2. McKay V, Cairns D, Gokhale D, Mountford R and Greenhalgh L: First report of somatic mosaicism for mutations in STK11 in four patients with Peutz-Jeghers syndrome. Fam Cancer 15: 57-61, 2016.

3. Jenne DE, Reomann H, Nezu J, Friedel W, Loff S, Jeschke R, Müller O, Back W and Zimmer M: Peutz-Jeghers syndrome is caused by mutations in a novel serine threonine kinase. Nat Genet 18: 38-43, 1998.
4. Hemminki A, Markie D, Tomlinson I, Avizienyte E, Roth S, Loukola A, Bignell G, Warren W, Aminoff M, Höglund P, et al: A serine/threonine kinase gene defective in Peutz-Jeghers syndrome. Nature 391: 184-187, 1998.

5. Wang Z, Ellis I, Zauber P, Iwama T, Marchese C, Talbot I, Xue WH, Yan ZY and Tomlinson I: Allelic imbalance at theLKB1 (STK11) locus in tumours from patients with Peutz-Jeghers' syndrome provides evidence for a hamartoma-(adenoma)-carcinoma sequence. J Pathol 188: 9-13, 1999.

6. Wang Z, Wu B, Mosig RA, Chen Y, Ye F, Zhang Y, Gong W, Gong L, Huang F, Wang X, et al: STK11 domain XI mutations: Candidate genetic drivers leading to the development of dysplastic polyps in Peutz-Jeghers syndrome. Hum Mutat 35: 851-858, 2014

7. Ylikorkala A, Avizienyte E, Tomlinson IP, Tiainen M, Roth S, Loukola A, Hemminki A, Johansson M, Sistonen P, Markie D, et al: Mutations and impaired function of LKB1 in familial and non-familial Peutz-Jeghers syndrome and a sporadic testicular cancer. Hum Mol Genet 8: 45-51, 1999.

8. Zhao RX and Xu ZX: Targeting the LKB1 tumor suppressor. Curr Drug Targets 15: 32-52, 2014.

9. Boudeau J, Sapkota G and Alessi DR: LKB1, a protein kinase regulating cell proliferation and polarity. FEBS Lett 546: 159-165, 2003.

10. Vaahtomeri K and Mäkelä TP: Molecular mechanisms of tumor suppression by LKB1. FEBS Lett 585: 944-951, 2011.

11. Korsse SE, Peppelenbosch MP and van Veelen W: Targeting LKB1 signaling in cancer. Biochim Biophys Acta 1835: 194-210, 2013.

12. Corradetti MN, Inoki K, Bardeesy N, DePinho RA and Guan KL: Regulation of the TSC pathway by LKB1: Evidence of a molecular link between tuberous sclerosis complex and Peutz-Jeghers syndrome. Gene Dev 18: 1533-1538, 2004.

13. Shaw RJ, Bardeesy N, Manning BD, Lopez L, Kosmatka M, DePinho RA and Cantley LC: The LKB1 tumor suppressor negatively regulates mTOR signaling. Cancer Cell 6: 91-99, 2004.

14. Zhou W, Marcus AI and Vertino PM: Dysregulation of mTOR activity through LKB1 inactivation. Chin J Cancer 32: 427-433, 2013.

15. Magnuson B, Ekim B and Fingar DC: Regulation and function of ribosomal protein S6 kinase (S6K) within mTOR signalling networks. Biochem J 441: 1-21, 2012.

16. Fenton TR and Gout IT: Functions and regulation of the $70 \mathrm{kDa}$ ribosomal S6 kinases. Int J Biochem Cell Biol 43: 47-59, 2011.

17. Meyuhas O and Dreazen A: Ribosomal protein S6 kinase: From TOP mRNAs to cell size. In: Progress in molecular biology and translational science. Hershey J (ed.): pp109-153, 2009.

18. Zoncu R, Efeyan A and Sabatini DM: mTOR: From growth signal integration to cancer, diabetes and ageing. Nat Rev Mol Cell Biol 12: 21-35, 2011.

19. Inoki K, Kim J and Guan K: AMPK and mTOR in cellular energy homeostasis and drug targets. Annu Rev Pharmacol Toxicol 52: 381-400, 2012.

20. Shaw RJ: LKB1 and AMP-activated protein kinase control of mTOR signalling and growth. Acta Physiol (Oxf) 196: 65-80, 2009.

21. Wei C, Amos CI, Zhang N, Wang X, Rashid A, Walker CL, Behringer RR and Frazier ML: Suppression of Peutz-Jeghers polyposis by targeting mammalian target of rapamycin signaling. Clin Cancer Res 14: 1167-1171, 2008.

22. van Veelen W, Korsse SE, van de Laar L and Peppelenbosch MP: The long and winding road to rational treatment of cancer associated with LKB1/AMPK/TSC/mTORC1 signaling. Oncogene 30: 2289-2303, 2011

23. Livak KJ and Schmittgen TD: Analysis of relative gene expression data using real-time quantitative PCR and the 2(-Delta Delta C(T)) method. Methods 25: 402-408, 2001.

24. Boardman LA, Couch FJ, Burgart LJ, Schwartz D, Berry R, McDonnell SK, Schaid DJ, Hartmann LC, Schroeder JJ, Stratakis CA and Thibodeau SN: Genetic heterogeneity in Peutz-Jeghers syndrome. Hum Mutat 16: 23-30, 2000.

25. Mehenni H, Resta N, Guanti G, Mota-Vieira L, Lerner A, Peyman M, Chong KA, Aissa L, Ince A, Cosme A, et al: Molecular and clinical characteristics in 46 families affected with peutz-jeghers syndrome. Dig Dis Sci 52: 1924-1933, 2007.

26. Boudeau J, Kieloch A, Alessi DR, Stella A, Guanti G and Resta N: Functional analysis of LKB1/STK11 mutants and two aberrant isoforms found in Peutz-Jeghers Syndrome patients. Hum Mutat 21: 172, 2003. 
27. Hemminki A, Tomlinson I, Markie D, Järvinen H, Sistonen $P$, Björkqvist AM, Knuutila S, Salovaara R, Bodmer W, ShibataD, et al: Localization of a susceptibility locus for Peutz-Jeghers syndrome to $19 \mathrm{p}$ using comparative genomic hybridization and targeted linkage analysis. Nat Genet 15: 87-90, 1997.

28. Sanchez-Cespedes M: A role for LKB1 gene in human cancer beyond the Peutz-Jeghers syndrome. Oncogene 26: 7825-7832, 2007.

29. Boudeau J, Baas AF, Deak M, Morrice NA, Kieloch A, Schutkowski M, Prescott AR, Clevers HC and Alessi DR: MO25alpha/beta interact with STRADalpha/beta enhancing their ability to bind, activate and localize LKB1 in the cytoplasm. EMBO J 22: 5102-5114, 2003.

30. Smith DP, Spicer J, Smith A, Swift S and Ashworth A: The Mouse Peutz-Jeghers Syndrome gene Lkbl encodes a nuclear protein kinase. Hum Mol Genet 8: 1479-1485, 1999.

31. Baas AF, Boudeau J, Sapkota GP, Smit L, Medema R, Morrice NA, Alessi DR and Clevers HC: Activation of the tumour suppressor kinase LKB1 by the STE20-like pseudokinase STRAD. EMBO J 22: 3062-3072, 2003.

32. Mehenni H, Gehrig C, Nezu J, Oku A, Shimane M, Rossier C, Guex N, Blouin JL, Scott HS and Antonarakis SE: Loss of LKB1 kinase activity in Peutz-Jeghers syndrome, and evidence for allelic and locus heterogeneity. Am J Hum Genet 63: 1641-1650, 1998.

33. Alessi DR, Sakamoto K and Bayascas JR: LKB1-dependent signaling pathways. Annu Rev Biochem 75: 137-163, 2006.

34. Miyoshi H, Nakau M, Ishikawa T, Seldin MF, Oshima M and Taketo MM: Gastrointestinal hamartomatous polyposis in Lkb1 heterozygous knockout mice. Cancer Res 62: 2261-2266, 2002.

35. Mehenni H, Resta N, Park JG, Miyaki M, Guanti G and Costanza MC: Cancer risks in LKB1 germline mutation carriers. Gut 55: 984-990, 2006

36. van Lier MGF, Westerman AM, Wagner A, Looman CW, Wilson JH, de Rooij FW, Lemmens VE, Kuipers EJ, Mathus-Vliegen EM and van Leerdam ME: High cancer risk and increased mortality in patients with Peutz-Jeghers syndrome. Gut 60: 141-147, 2011.
37. Shackelford DB, Vasquez DS, Corbeil J, Wu S, Leblanc M, Wu CL, Vera DR and Shaw RJ: mTOR and HIF-1alpha-mediated tumor metabolism in an LKB1 mouse model of Peutz-Jeghers syndrome. Proc Natl Acad Sci USA 106: 11137-11142, 2009.

38. Sanchez-Cespedes M, Parrella P, Esteller M, Nomoto S, Trink B, Engles JM, Westra WH, Herman JG and Sidransky D: Inactivation of LKB1/STK11 is a common event in adenocarcinomas of the lung. Cancer Res 62: 3659-3662, 2002.

39. Partanen JI, Nieminen AI, Mäkelä TP and Klefstrom J: Suppression of oncogenic properties of c-Myc by LKB1-controlled epithelial organization. Proc Natl Acad Sci USA 104: 14694-14699, 2007.

40. Lee JH, Koh H, Kim M, Park J, Lee SY, Lee S and Chung J: JNK pathway mediates apoptotic cell death induced by tumor suppressor LKB1 in Drosophila. Cell Death Differ 13: 1110-1122, 2006.

41. Zeng PY and Berger SL: LKB1 is recruited to the p21/WAF1 promoter by $\mathrm{p} 53$ to mediate transcriptional activation. Cancer Res 66: 10701-10708, 2006.

42. Karuman P, Gozani O, Odze RD, Zhou XC, Zhu H, Shaw R, Brien TP, Bozzuto CD, Ooi D, Cantley LC and Yuan J: The Peutz-Jegher gene product LKB1 is a mediator of p53-dependent cell death. Mol Cell 7: 1307-1319, 2001.

43. Hudson CC, Liu M, Chiang GG, Otterness DM, Loomis DC, Kaper F, Giaccia AJ and Abraham RT: Regulation of hypoxia-inducible factor 1alpha expression and function by the mammalian target of rapamycin. Mol Cell Biol 22: 7004-7014, 2002.

44. Jung CH, Jun CB, Ro SH, Kim YM, Otto NM, Cao J, Kundu M and Kim DH: ULK-Atg13-FIP200 complexes mediate mTOR signaling to the autophagy machinery. Mol Biol Cell 20: 1992-2003, 2009.

45. Moschetta M, Reale A, Marasco C, Vacca A and Carratu MR: Therapeutic targeting of the mTOR-signalling pathway in cancer: Benefits and limitations. Br J Pharmacol 171: 3801-3813, 2014. 